\title{
Cultura e Comprometimento Organizacional em Empresas Hoteleiras
}

\author{
Évora Mandim Ribeiro Naves \\ Marília Ferreira Dela Coleta
}

\section{ResUmo}

Trata-se de uma pesquisa de campo que analisa a relação dos tipos de cultura organizacional presentes em algumas empresas hoteleiras com a natureza do vínculo estabelecido entre indivíduo e organização. O campo de pesquisa foi um grupo de hotéis convencionais da cidade de Uberlândia. O grupo de variáveis independentes do modelo proposto compreendia variáveis pessoais/profissionais e os tipos de cultura organizacional. A variável dependente era representada pelas três dimensões do comprometimento. Utilizou-se, além da estatística descritiva, análise de correlações e regressão múltipla. Os resultados indicaram a existência de relação dos tipos de cultura com os tipos de comprometimento. A cultura de função é o principal preditor do comprometimento normativo e instrumental e a cultura de tarefa é o principal preditor do comprometimento afetivo.

Palavras-chaves: hotelaria; cultura organizacional; comprometimento organizacional.

\begin{abstract}
The present study is a field research that tried to investigate the relationships between the types of organizational culture and organizational commitment in some hotels. The research was accomplished with a group of conventional hotels from Uberlandia, Brazil. The group of independent variables was formed by personal/professional and types of organizational culture. The dependent variable was composed by types of organizational commitment. Besides descriptive statistics, analysis of correlations and multiple regression appear on the study. The results showed the existence of relationships between the types of organizational culture and the commitment types. It was identified that the function culture is the main predictor of the normative and continuance entails and the culture of task of the affective commitment.
\end{abstract}

Key words: business hotel; organizational culture; organizational commitment. 


\section{INTRODUÇÃO}

O turismo é hoje um campo de investimento que vem ganhando importância cada vez maior no contexto do desenvolvimento socioeconômico. Basta ver que algumas cidades que, há poucos anos, não figuravam no mapa nacional do turismo, hoje constituem fortes centros receptores, impulsionando, desta forma, sua arrancada para o desenvolvimento. Dentro deste contexto, a indústria hoteleira não pode ser considerada atividade marginal, mas elemento estratégico para a política de desenvolvimento turístico de uma região.

Por se tratar de empresa prestadora de serviços, o diferencial da qualidade hoteleira não está somente na qualidade objetiva, mas também na qualidade subjetiva, que se expressa diretamente pelo provedor do serviço, representada pela atitude e comprometimento dos funcionários com a empresa.

Assim, para as empresas hoteleiras, fica clara a importância em propiciar uma cultura organizacional que promova incessantemente a busca do comprometimento dos seus clientes internos, pois somente a partir de funcionários dedicados e dispostos a vestir a camisa será possível à organização oferecer serviços com qualidade e que superem as expectativas dos clientes. Emerge, então, um questionamento: a natureza do vínculo estabelecido entre indivíduo e organização é influenciada por quais tipos de cultura organizacional?

O comprometimento organizacional, em termos gerais, pode ser entendido como o envolvimento com a organização que incita a realizar um esforço considerável em prol da empresa, sendo este afetado pela natureza do vínculo: afetiva, instrumental ou normativa.

Dentro deste panorama, torna-se importante para a indústria hoteleira identificar a natureza do vínculo predominante e a relação dos tipos de cultura organizacional percebidos pelos funcionários com a natureza do comprometimento organizacional por eles desenvolvido.

Com o objetivo de contribuir para esta área do conhecimento, foi desenvolvida uma pesquisa de campo, que procurou identificar a existência de possíveis relações dos tipos de cultura organizacional com a natureza do comprometimento organizacional em hotéis convencionais da cidade de Uberlândia, MG.

Este trabalho, em primeiro lugar, realiza uma reflexão conceitual sobre o construto cultura, abordando mais especificamente a teoria dos Deuses da Administração 
de Handy (1994), instrumentalizado por Gomide Jr. e Martins (1997). Em seguida referencia-se o construto comprometimento organizacional, abordando o conceito, os principais enfoques e o modelo das três dimensões de Meyer e Allen (1991), tomado como objeto de estudo, utilizando-se as escalas multifatoriais validadas por Medeiros (1997). Posteriormente apresentam-se os resultados da pesquisa realizada nos hotéis na cidade de Uberlândia e, finalmente, são feitas algumas considerações que podem servir de indicações para futuras pesquisas.

O interesse em estudar a relação entre os construtos cultura organizacional e comprometimento organizacional nas empresas hoteleiras, deve-se à recentidade dos temas e à tendência de se pesquisar fatores macroorganizacionais como antecedentes do comprometimento organizacional. A exemplo disto, tem-se: Bandeira (1999) contribuiu investigando a relação da política de recursos humanos com os padrões de comprometimentos presentes em uma empresa pública; Oliveira (1997) investigou os valores e mitos organizacionais como determinantes do vínculo afetivo com a organização; Martins (1998) procurou demonstrar as relações que podem existir entre configurações de poder e comprometimento, num contexto de mudança organizacional; Borges-Andrade e Pilati (1998) procuraram medir as percepções de imagens organizacionais definidas por Morgan e suporte organizacional, a partir da percepção de funcionários; e Bastos e Rocha (1999) procuraram relacionar os padrões de comprometimento, baseados no modelo das três dimensões de Meyer e Allen, com a visão sobre os impactos dos programas de mudança. Além disso, os autores Gomide Jr. e Martins (1997), em seus trabalhos, sugerem como pauta de pesquisas futuras a correlação entre cultura organizacional e critérios comportamentais como comprometimento organizacional.

\section{Cultura Organizacional}

A utilização do conceito de cultura organizacional na literatura de administração se estabeleceu a partir da década de 80 , embora ele antes já fosse empregado e perdura até os dias atuais. São grandes os números de propostas conceituais encontradas na literatura, que atestam as divergências nos rumos da pesquisa.

Os teoristas organizacionais e gestores têm usado uma variedade de termos como metáforas ou imagens, para limitar o cerco e diferenciar as categorias de experiências que se referem ao estilo das organizações (Smircich, 1983).

De modo geral, pode-se dizer que cultura organizacional representa uma energia social que instiga os membros a agir, fornecendo significado e direção, e também um mecanismo de controle, aprovando informalmente ou proibindo 
comportamentos. Elementos como ritos, mitos, valores, crenças, pressupostos, normas, regras, símbolos e emoções são alguns dos componentes integrantes deste construto. Hofstede (1997) define cultura como "a programação coletiva da mente que distingue os membros de uma organização dos de outra". Nesse sentido, a corrente que estuda cultura organizacional como tipos ou classificações se aplica perfeitamente bem ao propósito do trabalho.

Segundo Freitas (1991) são poucos os pesquisadores que se lançaram a responder à pergunta "que tipos de culturas organizacionais existem?". As classificações ou tipologias surgidas não correspondem à realidade da empresa pesquisada em todo os seus matizes, mas nos permitem classificar ou categorizar a empresa sob determinado prisma (Freitas, 1991). Assim surgiram na literatura os Deuses da Administração de Handy (1994), as categorizações de Deal e Kennedy (1982), entre outras.

\section{Os Deuses da Administração de Charles Handy}

Para Handy (1994), a administração de organizações não é ciência exata, mas processo criativo e político, no qual cada organização se diferencia das outras em função das características de sua administração, padrões, modelos e orientações que podem ser identificados. Embora não defina cultura organizacional, o autor propõe quatro tipos de culturas, que podem ser discernidas nas empresas, caracterizando quatro diferentes maneiras de administrar, cada uma simbolizada por um deus grego. O autor afirma que cada cultura opera a partir de suposições distintas sobre a base do poder e da influência, sobre o que motiva as pessoas, como elas pensam, como elas aprendem e como as situações e os comportamentos podem ser mudados.

\section{Cultura Zeus}

O primeiro deus grego, Zeus, representa a Cultura do Clube ou cultura do Poder. Esta cultura é caracterizada pela existência de um poder central, prevalecendo os desejos e decisões das fontes centrais. Existem poucas regras e procedimentos documentados, o controle é exercido pela figura central. As decisões são tomadas em grande parte com base nos resultados de experiências passadas e não com base em razões processuais puramente lógicas (Freitas, 1991). O poder é exercido por meio da confiança e afinidade entre as pessoas; os esforços dos subordinados são recompensados com atribuições de maiores responsabilidades e a seleção de pessoal é baseada em vínculos pessoais. O treinamento é informal no cargo. Como vantagens da cultura Zeus pode-se destacar o dinamismo, pois reage rapidamente às demandas externas. Entretanto as culturas de poder podem sofrer desafetos pela equipe. 


\section{Cultura Apolo}

A segunda cultura proposta por Handy (1994) é representada pela figura do deus grego Apolo, deus da ordem, e identifica a Cultura da Função. "Esta cultura é caracterizada por ser tipicamente uma burocracia, onde o indivíduo é encarado como parte integrante do fluxo de produção e dele espera-se que cumpra o seu dever" (Gomide Jr., 1999, p.76).

As funções são muito bem definidas e especializadas, sendo prescritas num conjunto de regras e procedimentos. A estabilidade e a previsibilidade são assumidas e encorajadas. Os indivíduos desta cultura são parte da máquina: "é irrelevante que o indivíduo tenha um nome, um número serviria da mesma forma. É bem inconveniente que ele tenha uma personalidade, porque ele poderia ser tentado a expressar essa personalidade na função, e assim alterá-la" (Handy, 1994). Com relação à estrutura organizacional, há um organograma claro e bem definido, sendo o poder exercido e centrado no topo da estrutura. $\mathrm{O}$ treinamento é do tipo formal voltado para suprimento das necessidades de curto prazo do cargo (Gomide Jr., 1999). Na cultura Apolo a finalidade da ação de coordenar é inspecionar, assegurando que as regras e os procedimentos sejam cumpridos. São culturas apropriadas, em que a economia de escala e a perícia técnica são mais importantes que a flexibilidade e a inovação.

\section{Cultura Atena}

O terceiro tipo, a cultura da tarefa, é representado pela deusa Atena, uma jovem guerreira, patrona de Ulisses, aquele arqui-solucionador de problemas, dos artesãos e dos líderes pioneiros. A cultura é caracterizada pela liderança consensual, pela meritocracia, recompensando-se o talento, a criatividade, a inovação e o trabalho em equipe. O desempenho é avaliado em termos de resultados alcançados. Em Atena o treinamento é voltado para solução de problemas, para o autodesenvolvimento. Os indivíduos são pensados como humanos de recursos, em vez de recursos humanos, sendo vistos como pessoas responsáveis pelos seus destinos finais. Na cultura de tarefa, a obediência é substituída pela concordância. As discussões em grupos e a argumentação são estimuladas. Pode-se dizer que a cultura da tarefa é flexível e adaptável. As equipes são formadas para resolverem um projeto. A cultura de tarefa tende a se desenvolver bem, quando o clima da organização é agradável, quando o produto é importante e o cliente está acima de tudo. 


\section{Cultura Dionísio}

O quarto tipo de cultura proposto por Handy (1994) é a cultura existencial representada pela figura do deus Dionísio, que representa a ideologia existencial entre os deuses. É o deus do indivíduo, da liberdade de espírito. Nas outras três culturas o indivíduo é subordinado à organização. Nesta cultura a organização se caracteriza pela preservação da identidade e liberdade individual do empregado, por privilegiar o talento e a habilidade e pelo fato de o empregado administrar seu próprio destino. As organizações de Dionísio são organizações democratas no seu sentido puro. $\mathrm{O}$ administrador governa com o consentimento dos governados e não com a autoridade delegada pelo proprietário. Nas organizações de Dionísio a qualidade do trabalho está acima de tudo. A cultura de Dionísio é excelente, onde o recurso crucial da organização é o talento, ou a habilidade do indivíduo. É a cultura preferida pelos profissionais liberais, artistas e artesãos, pois eles podem preservar sua própria liberdade sem sentir-se como propriedade de alguém.

Optou-se por pesquisar cultura organizacional, a partir da fundamentação teórica de Handy (1994), por tratá-la como uma variável organizacional, representando o seu conjunto de práticas administrativas, sendo possível relacioná-la à natureza do vínculo estabelecido entre indivíduo e organização.

\section{Comprometimento Organizacional}

Assim como cultura, o tema comprometimento organizacional tem sido objeto de estudo de vários pesquisadores nas últimas décadas, objetivando auxiliar as organizações a encontrarem estratégias de gerenciamento do seu pessoal que o tornem mais satisfeito, mais envolvido com a empresa e, por conseguinte, mais produtivo.

Comprometimento é estudado em função dos focos ou alvos e das bases ou natureza do vínculo. São inúmeros os alvos de vínculo que o trabalhador tem: a organização, o grupo, o sindicato, os valores, a profissão, o trabalho etc.; assim como são inúmeras as naturezas destes vínculos: afetiva, instrumental, normativa etc. Vale ressaltar ainda que, dentro de cada foco (alvo), especialmente com a organização e o sindicato, existem abordagens diferenciadas quanto às bases (naturezas).

São cinco as correntes de pesquisa sobre comprometimento: afetivo-atitudinal, instrumental (contínua ou side-bet), normativa, sociológica e comportamental. Embora existam várias vertentes que abordem o construto comprometimento 
organizacional, elas compartilham a premissa de que o vínculo existe e é inevitável, significando o desejo de permanecer na organização, de exercer suas atividades, de acreditar e aceitar os objetivos e valores organizacionais.

\section{Enfoque Afetivo-Atitudinal}

A linha de pesquisa que considera a natureza do vínculo como afetivo-atitudinal prevalece entre os pesquisadores. Suas bases se fundamentam nas teorias de Etzioni (1975), sendo esta abordagem mais tarde aprimorada com os trabalhos de Mowday, Steers e Porter (1979). Para os autores, estar comprometido com a organização significa a identificação e o envolvimento com a organização. O indivíduo comprometido realiza um esforço considerável em prol da organização. Assim, eles o caracterizam como: (1) uma forte crença e aceitação dos objetivos e valores da organização; (2) uma tendência a manifestar um esforço considerável em favor da organização; e (3) a manifestação de um forte desejo de permanecer como membro da organização.

A partir desta definição, verifica-se que indivíduos com elevado grau de comprometimento demonstram uma internalização dos valores e objetivos da organização e, por conseguinte, tendem a exercer um esforço considerável em favor dela. Sob esse ponto de vista, os indivíduos assumem uma postura ativa. Estão dispostos a se empenhar ao máximo, contribuindo de forma positiva para a empresa.

\section{Enfoque Instrumental}

O enfoque instrumental constitui o segundo grande referencial teórico adotado nas pesquisas sobre o tema, cujas denominações são: calculativo, continuação, side-bets (Bastos, 1994). Esta corrente originou-se nos trabalhos de Becker (1960), traduzindo-o como função da percepção do trabalhador, quanto às trocas estabelecidas com a organização. Segundo o autor, o indivíduo permanece na organização devido a custos e benefícios associados a sua saída. Em outras palavras, o empregado irá optar por permanecer na empresa enquanto perceber benefícios nessa escolha. No momento em que perceber um déficit em relação aos retornos recebidos, sua escolha provavelmente será abandonar a empresa.

O comprometimento instrumental foi operacionalizado pelas escalas desenvolvidas, num primeiro momento, por Ritzer e Trice (1969) e, posteriormente, por Hrebiniak e Alluto (1972). 


\section{Enfoque Normativo}

A corrente de pesquisa normativa é ancorada nos trabalhos de Wiener (1982) e Wiener e Vardi (1990), influenciada pelas contribuições de Etzioni (1975). Segundo a corrente normativa, o comprometimento é visto como o conjunto de pressões normativas, internalizadas para que o indivíduo se comporte de acordo com os objetivos e metas da organização. O enfoque normativo procura trabalhar o plano organizacional por meio da análise da cultura e do lado individual, por meio dos processos motivacionais. Parte do pressuposto de que o comportamento do indivíduo é conduzido de acordo com o conjunto de normas que ele assume internamente.

Wiener e Vardi (1990) defendem que a cultura organizacional é capaz de atuar junto aos empregados com o intuito de envolvê-los nos ideais da organização, permanecendo esta influência estável no corpo dos funcionários. É importante destacar que esta adesão vai depender dos valores e normas partilhados e do que os membros acreditam ser a conduta ética e moral.

Uma terceira corrente que merece destaque e ampara os estudos desta pesquisa é o enfoque das três dimensões de Meyer e Allen (1991).

\section{O Enfoque das Três Dimensões de Meyer e Allen}

A conceitualização do comprometimento organizacional em três dimensões, afetivo, instrumental e normativo, foi decorrente da análise de variância (ANOVA) realizada por Meyer e Allen (1991). Nesta análise eles concluíram que as escalas propostas por Ritzer-Trice e Hrebiniak-Alluto não mediam o comprometimento instrumental, conceitualizado por Becker (1960), pois apresentava maior correlação com o componente afetivo. Com isso desenvolveram dois questionários, um para medir o comprometimento afetivo, Affective Commitment Scale (ACS), e outro para medir o comprometimento instrumental, Continuance Commitment Scale (CCS).

Mais tarde, McGee e Ford (1987), aplicando as escalas desenvolvidas por Meyer e Allen (1991), utilizando a análise fatorial, verificaram o surgimento de uma nova dimensão do comprometimento, denominada normativa, composta de itens que refletiam o sacrifício pessoal do empregado na hipótese de deixar a organização.

A conceitualização da dimensão normativa está apoiada no trabalho de Wiener (1982). Posteriormente, Meyer e Allen (1990) incorporaram à nova dimensão e conceitualizaram o comprometimento organizacional em função das três dimensões: 
. comprometimento como apego afetivo com a organização.

. comprometimento percebido em função dos custos associados a deixar a organização.

. comprometimento como obrigação de permanecer na organização.

Meyer e Allen (1990, p.3) caracterizam os três tipos de vínculos entre indivíduos e organização da seguinte forma: empregados com forte comprometimento afetivo permanecem na organização, porque eles querem; aqueles com comprometimento instrumental permanecem porque eles precisam; e aqueles com comprometimento normativo permanecem porque eles sentem que são obrigados.

Segundo os autores, estes três tipos de bases estariam presentes no vínculo indivíduo-organização, porém com intensidades diferenciadas.

Esta abordagem múltipla está presente nas agendas de pesquisadores tanto brasileiros (Medeiros, 1997; Bandeira, 1999; Bastos, 1999) como estrangeiros (Clugston, 2000).

\section{Comprometimento Organizacional e Cultura Organizacional}

Para alguns teóricos em comprometimento organizacional, a cultura organizacional é um importante antecedente do comprometimento (Meyer e Allen, 1991; Wiener apud Clugston, 2000).

A cultura organizacional, para Oliveira (1997), exerce o papel de produzir membros organizacionais mais comprometidos, influenciando no comportamento, à medida que se criam padrões e que são internalizados pelos indivíduos.

De acordo com Meyer e Allen (1991), cada uma das bases do comprometimento (afetivo, instrumental e normativo) é afetada por diferentes antecedentes. O comprometimento normativo é inicialmente influenciado pelas orientações sociais e culturais de cada empregado (Wiener, 1982; Meyer e Allen, 1991).

Contrariando a tendência de pesquisadores estrangeiros de investigar os aspectos microorganizacionais como preditores do comprometimento, evidencia-se o trabalho de Clugston (2000), que procura relacionar as quatro dimensões da cultura de Hofstede com as três dimensões do comprometimento de Meyer e Allen (1991), tendo como focos de investigação a organização, a supervisão e o grupo de trabalho. 
Assim, o que caracteriza este trabalho é acompanhar a tendência de tratar o comprometimento no modelo de três dimensões e relacioná-lo à cultura organizacional.

\section{Metodologia}

Trata-se de uma pesquisa de campo que procurou estudar a relação entre os tipos de cultura organizacional, propostos por Handy (1994), e as três dimensões do comprometimento de Meyer e Allen (1991).

Como variável dependente colocou-se o comprometimento organizacional e as suas três dimensões: afetiva, normativa e instrumental. O grupo de variáveis independentes do modelo testado foi a cultura organizacional e as variáveis biográficas e profissionais.

O construto cultura organizacional foi operacionalizado segundo a teoria de Handy (1994), a partir do instrumento validado por Gomide Jr. e Martins (1997). Todos os itens foram respondidos com base em uma escala do tipo Likert, de cinco pontos, com as opções de resposta: $1=$ discordo totalmente a $5=$ concordo totalmente. Trata-se de uma escala unifatorial com 28 afirmações, constituída assim: (1) escala de cultura clube, composta de seis itens; (2) escala de cultura de função, com dez itens; (3) escala de cultura de tarefa, com cinco itens; e (4) escala de cultura de pessoa, com sete itens.

A variável dependente comprometimento organizacional foi operacionalizada segundo a teoria das três dimensões de Meyer, Allen e Smith (1993), a partir do instrumento validado por Medeiros (1997) no Brasil. Trata-se de uma escala multifatorial com 18 afirmações, sendo os seis primeiros indicadores de comprometimento afetivo, os seis seguintes indicadores de comprometimento instrumental e os seis últimos indicadores de comprometimento normativo. Para esta escala, foi utilizado igualmente o formato Likert com cinco níveis de resposta.

O campo de pesquisa foi um grupo de hotéis convencionais da cidade de Uberlândia, MG, incluindo nesta categoria os hotéis residência. A escolha dos hotéis foi intencional, e os critérios foram: ser classificados como muito confortável e médio conforto, pela classificação do Guia Quatro Rodas, e que tivessem mais de 100 UH's.

Um total de 223 sujeitos foram pesquisados de um universo de 251 funcionários, de quatro hotéis, sendo três hotéis convencionais e um hotel residência. 
Participaram todos os funcionários registrados em carteira e que possuíssem escolaridade correspondente pelo menos ao primeiro grau incompleto.

Os questionários foram aplicados em grupo de dez pessoas, que responderam individualmente, em local especialmente reservado para este fim.

\section{Análise e Discussão dos Resultados}

O trabalho realizado consistiu, fundamentalmente, em identificar os tipos de cultura organizacional que predizem cada uma das dimensões do comprometimento organizacional. Essa questão central pode ser concluída graças às técnicas estatísticas de correlação linear (r) de Pearson e pela técnica de análise de regressão múltipla, método stepwise.

Pode-se verificar, a partir dos resultados da Tabela 1, que as variáveis que apresentaram uma relação de linearidade direta com a dimensão normativa foram a idade, a percepção do treinamento como oportunidade de desenvolvimento, o nível de expectativas atendidas nos primeiros meses de trabalho e o nível de escolaridade, que se relacionou inversamente. As culturas que apresentaram uma relação de linearidade com a dimensão normativa do comprometimento foram as seguintes: existencial (EPCE), função (EPCF) e, por último, a cultura da tarefa (EPCT).

\section{Tabela 1: Coeficientes de Correlação das Variáveis Culturais, Pessoais e da Empresa com as Três Dimensões do Comprometimento Organizacional}

\begin{tabular}{l|c|c|c}
\hline \hline & NORMATIVA & INSTRUMENTAL & AFETIVA \\
\hline EPCC & $-0,098$ & 0,037 & $-0,223^{* *}$ \\
\hline EPCT & $0,321^{* *}$ & 0,073 & $0,447^{* *}$ \\
\hline EPCF & $0,397^{* *}$ & $0,175^{* *}$ & $0,314^{* *}$ \\
\hline EPCE & $0,409^{* *}$ & $0,147^{*}$ & $0,292^{* *}$ \\
\hline Idade & $0,238^{* *}$ & $0,151^{*}$ & 0,10 \\
\hline Escolaridade & $-0,196^{* *}$ & $-0,162^{*}$ & 0,10 \\
\hline Salário das expectativas & 0,045 & $-0,083$ & $0,234^{* *}$ \\
\hline $\begin{array}{l}\text { Nível } \\
\text { atendidas }\end{array}$ & $0,211^{* *}$ & $0,165^{*}$ & $0,273^{* *}$ \\
\hline $\begin{array}{l}\text { Avaliação da oportunidade de } \\
\text { treinamento }\end{array}$ & $0,257^{* *}$ & 0,065 & $0,375^{* *}$ \\
\hline \hline
\end{tabular}

Fonte: dados da pesquisa.

* Correlação é significativa ao nível de 0,05 .

** Correlação é significativa ao nível de 0,01 . 
Steers (apud Medeiros, 1997, p.79) encontrou resultados semelhantes, em que o nível educacional é inversamente relacionado ao comprometimento: "quando os empregados possuem altos níveis educacionais, deve ser mais difícil às organizações proporcionarem recompensas satisfatórias".

Em relação à dimensão instrumental do vínculo, as mulheres apresentaram-se mais comprometidas instrumentalmente do que os funcionários do sexo masculino. O salário, embora não tenha demonstrado uma relação de linearidade com a dimensão instrumental, mostrou correlação no sentido inverso. Tal resultado vem corroborar os estudos de Mathieu e Zajac (1990), que apontam o comprometimento puramente instrumental para uma relação negativa com a variável salário, enquanto os comprometimentos com predomínio afetivo e normativo, que não estão associados a componentes instrumentais, estão relacionados positivamente ao salário. A variável cultura de função (EPCF) apresentou uma relação de linearidade com essa dimensão.

Para a natureza do vínculo afetivo as variáveis que apresentaram uma relação direta foram o salário, o nível das expectativas do trabalho atendidas nos primeiros meses de trabalho e a avaliação do treinamento como oportunidade de desenvolvimento. Além disso, verificou-se que o grupo de indivíduos casados apresentou maior grau da dimensão afetiva do comprometimento que os grupos de solteiros ou sozinhos. Mathieu e Zajac (1990) encontraram uma leve correlação positiva entre indivíduos casados e o comprometimento organizacional. As culturas que apresentaram uma relação de linearidade com essa dimensão, obedecendo à ordem de importância, foram: a cultura de tarefa (EPCT), a de função (EPCF) e por último a cultura existencial (EPCE). Já a cultura clube (EPCC) apresentou uma relação de linearidade inversa com a natureza do vinculo afetivo.

O trabalho de Bandeira (1999) comprova a relação significativa direta da oferta de treinamento como oportunidade de desenvolvimento com a dimensão afetiva e normativa do vínculo. $O$ fato de a empresa reconhecer a necessidade de qualificar os funcionários e investir no seu desenvolvimento profissional em função da carência do setor por mão-de-obra qualificada, gera no indivíduo um sentimento de lealdade e afetividade para com a empresa, favorecendo sua permanência e engajamento com as ações da empresa.

\section{Preditores das Três Dimensões do Comprometimento Organizacional}

Os resultados da análise de regressão múltipla, conforme ilustrado na Tabela 2, demonstraram que o principal preditor da dimensão normativa foi o estilo de cultura de função (EPCF), contribuindo com $38,6 \%$; em seguida a idade, contri- 
buindo com 18,8\%. Estas duas variáveis independentes mostraram-se preditoras, explicando em conjunto $20,3 \%$ de sua variância.

Tabela 2: Resultados da Análise de Regressão Múltipla para as Três Dimensões do Comprometimento

\begin{tabular}{|c|c|c|c|c|c|}
\hline Variável dependente & Variável independente & Coeficiente padronizado Beta & Sig. & $\mathrm{R}$ & $\mathrm{R}^{2}$ \\
\hline \multirow[b]{2}{*}{ NORMATIVA } & EPCF & 0,386 & 0,0001 & \multirow[b]{2}{*}{0,451} & \multirow[b]{2}{*}{0,203} \\
\hline & IDADE & 0,188 & 0,012 & & \\
\hline INSTRUMENTAL & EPCF & 0,175 & 0,010 & 0,175 & 0,031 \\
\hline \multirow{4}{*}{ AFETIVA } & EPCT & 0,351 & 0,0001 & \multirow{4}{*}{0,588} & \multirow{4}{*}{0,346} \\
\hline & EXPCTAT & 0,190 & 0,003 & & \\
\hline & SALARIO & 0,189 & 0,002 & & \\
\hline & AVTRE & 0,172 & 0,013 & & \\
\hline
\end{tabular}

Fonte: dados da pesquisa.

Para a dimensão afetiva do comprometimento organizacional, os principais preditores foram: cultura de tarefa (EPCT), contribuindo com 35,1\%; em seguida a expectativa atendida em relação à empresa nos primeiros meses de trabalho (EXPCTAT), contribuindo com 19,0\%; salário com 18,9\% e a variável percepção da avaliação do treinamento como oportunidade de desenvolvimento (AVTRE), com 17,2\%. Estas quatro variáveis explicaram em conjunto 34,6\% da variância da dimensão afetiva. Os estudos realizados por Oliveira e Gomide Jr. (1997) apontaram a cultura de tarefa como preditora do comprometimento afetivo e da intenção de rotatividade.

Verificou-se que o único preditor da dimensão instrumental do comprometimento organizacional foi a percepção da cultura de função (EPCF), que contribuiu com $17,5 \%$, explicando apenas $3,1 \%$ da variável independente.

Os resultados apontados anteriormente contrariam os trabalhos de Mathieu e Zajac (1990), que realizaram uma meta-análise sumariando os resultados das pesquisas sobre antecedentes de comprometimento organizacional, na qual encontraram correlações praticamente nulas entre características organizacionais e comprometimento.

\section{Conclusóes e Recomendaçóes}

Este estudo procurou demonstrar as relações entre os tipos de cultura organizacional e as três dimensões do comprometimento organizacional.

Concluiu-se que a cultura de função está relacionada ao comprometimento 
organizacional do tipo normativo, isto é, o funcionário se engaja nas ações da empresa porque se sente obrigado. Já a cultura de tarefa está relacionada diretamente ao vínculo de natureza afetiva. Isto significa dizer que tal cultura proporciona sentimentos de afetividade do indivíduo em relação à empresa, favorecendo sua permanência e seu envolvimento com as ações da organização. Talvez este resultado possa ser explicado pelas próprias características da cultura, em que o trabalho em equipe, a criatividade e a valorização das habilidades humanas são reconhecidas e recompensadas, sendo o funcionário visto como humanos de recursos, em vez de recursos humanos.

Motta (apud Paiva, 1993) corrobora o exposto, ao analisar a participação direta do trabalhador nas tarefas, afirmando que a democratização nas organizações modernas, a cooperação, a difusão de informações e a motivação no desempenho acarretam um comprometimento com a empresa.

Assim, na indústria hoteleira, onde o acolhimento interfere significativamente no fluxo de turistas (Avena, 2001), torna-se importante saber administrar o comprometimento dos funcionários, sobretudo favorecendo o predomínio das dimensões afetivas e normativas do vínculo indivíduo-organização.

A dimensão afetiva deve estar presente para proporcionar a espontaneidade nas ações e a atitude positiva. O indivíduo faz ou age daquela forma porque gosta, sente prazer em fazer, em servir. Por outro lado, a dimensão normativa sustentará o vínculo afetivo, no momento em que são amparados pelas regras, normas e procedimentos estabelecidos pela empresa.

Sabe-se, a partir deste trabalho, que na hotelaria o favorecimento de uma cultura que promova a participação do funcionário, o trabalho em grupo, o treinamento objetivando o crescimento profissional, juntamente com uma remuneração justa, propiciam o engajamento afetivo. Aliado a isto, a idade e a cultura, em que os papéis são bem definidos, proporcionam a dimensão normativa do vínculo.

Relacionar cultura organizacional e os múltiplos comprometimentos na hotelaria amplia o campo de investigação e contribui, de forma significativa, para um setor tão importante, porém esquecido no campo do comportamento organizacional.

Devem ser consideradas, também, algumas limitações do estudo. A amostra ficou limitada a um grupo de hotéis de uma cidade de porte médio, localizada no Triângulo Mineiro, com sua cultura particular, possivelmente influenciando a cultura destas organizações, mesmo naquelas não originárias da região.

Outro aspecto metodológico a ser considerado ao se observar estes resultados, diz respeito ao fato de que são as avaliações e percepções dos funcionários dos 
hotéis que estão sendo analisadas, por meio de suas respostas ao instrumento para medida da cultura organizacional, o que é diferente de se efetuar uma análise direta sobre os métodos, políticas e práticas organizacionais. Do mesmo modo como o funcionário avalia os indicadores dos diferentes tipos de cultura nas empresas hoteleiras pesquisadas, também ele avalia a natureza de seu de vínculo. Assim pretende-se deixar claro que os resultados reportados neste trabalho indicam a relação entre o comprometimento e a cultura organizacional, sendo ambos avaliados sob a ótica do empregado. Seria interessante estender esta pesquisa a uma amostra maior, relacionando as dimensões culturais de Hofsted (1997) aos tipos culturais propostos por Handy (1994) e às três dimensões do comprometimento organizacional de Meyer e Allen (1991).

Além do exposto aqui, nenhuma investigação se encontra concluída em termos definitivos. É necessário dar continuidade a esta linha de pesquisa e atualizar constantemente os conhecimentos neste campo, visando a obter maior satisfação no trabalho por parte dos funcionários e maior eficácia na prestação de serviços pelo setor de hotelaria

\section{ReferênCias Bibliográficas}

AVENA, B. M.

Acolhimento de qualidade: fator diferenciador para o incremento do turismo. Turismo em Análise, v. 12, n. 1, p. 20-29, maio 2001.

BANDEIRA, M. L.;

MARQUES, A. L.;

VEIGA, R. T.

A ECT na trilha da modernidade: políticas de recursos humanos influenciando múltiplos comprometimentos. In: ENCONTRO ANUAL ANPAD, 23., 1999, Foz do Iguaçu. Anais... Foz do Iguaçu: ANPAD, 1999. 1 CD-ROM.

BASTOS, A. V. B.

\section{Comprometimento no trabalho:}

a estrutura dos vínculos trabalhador com a organização, a carreira e o sindicato. Brasília, 1994. 294 f. Tese (Doutorado em Psicologia) - Instituto de Psicologia, Universidade de Brasília.

BASTOS, A. V. B.; ROCHA, A.

$\mathrm{S}$ de C. Comprometimento do empregado e contextos organizacionais em mudança: o caso do Banco do Brasil. In: ENCONTRO ANUAL ANPAD, 23., 1999, Foz do Iguaçu. Anais... Foz do Iguaçu: ANPAD, 1999. 1 CD-ROM. 
BECKER, H. S.

Notes on the concept of commitment. The American Journal of Sociology, v. 66, n. 1, p. 32-40, 1960.

BORGES-ANDRADE, J.;

PILATI, R.

Comprometimentos atitudinal e comportamental: relações com suporte e imagem nas organizações. In: ENCONTRO ANUAL ANPAD, 22., 1998. Foz do Iguaçu. Anais... Foz do Iguaçu: ANPAD, 1998. 1 CD-ROM.

\section{CLUGSTON, M.}

Does cultural socialization predict multiple bases and foci of commitment? Journal of Management, Jan. 2000 . Disponível em: <http:// www.findarticles.com $>$ Acesso em: 20 nov. 2001.

DEAL, T. E.;

KENNEDY, A. A.

Corporate cultures: the rites and rituals of corporate life. Reading, MA: Addison-Wesley, 1982.

ETZIONE, A.

Análise comparativa de organizações complexas: sobre o poder, o engajamento e seus correlatos. Rio de Janeiro: Zahar, 1975.

FREITAS, M. E. de.

Cultura organizacional: formação, tipologias e impacto. São Paulo: Makron Books, 1991.
GOMIDE JR., S.

Antecedentes e conseqüientes das percepções de justiça no trabalho. Brasília, 1999. $140 \mathrm{f}$. Tese (Doutorado em Psicologia) Instituto de Psicologia, Universidade de Brasília.

GOMIDE JR., S.;

MARTINS, M. do C.

Os deuses da administração: construção e validação de quatro escalas para medida de cultura organizacional. Psicologia: Teoria e Pesquisa, v. 13, n. 3, p. 311316, set./dez. 1997.

HANDY, C.

Deuses da administração: como enfrentar as constantes mudanças da cultura empresarial. São Paulo: Saraiva, 1994.

HOFSTEDE, G.

Culturas e organizações: compreender a nossa programação mental. Lisboa: Silabo, 1997.

HREBINIAK, L. G.;

ALLUTO, J. A.

Personal and role-related factors in the development of organizational commitment. Administrative Science Quaterly, v. 17, p. 555-573, 1972.

MARTINS, J. L.

Poder e comprometimento organizacional. Brasília, 1998. 99 f. Dissertação (Mestrado em Psicologia) - Instituto de Psicologia, Universidade de Brasília. 
MATHIEU, J. E.; ZAJAC, D. M.

A review and meta-analysis of the antecedents, correlates and consequences of organizational commitment. Psychological Bulletin, v. 108, p. 177/179, 1990.

MCGEE, G. W.;

FORD, R. C.

Two (or more?) dimensions of organizational commitment: reexamination of the affective and commitment scales. Journal of Applied Psychology, v. 72, p. 638-642, 1987.

\section{MEDEIROS, C. A.}

Comprometimento organizacional, características pessoais e performance no trabalho: um estudo dos padrões de comprometimento organizacional. Natal, 1997. 111 f. Dissertação (Mestrado em Administração) Programa de Pós-Graduação em Administração, Universidade Federal do Rio Grande do Norte.

MEYER, J. P.;

ALLEN, N. J.

A three-component conceptualization of organizational commitment. Human Resource Management Review, v. 1, p. 61-89, 1991.

MEYER, J. P.;

ALLEN, N. J.;

GELLATLY, I. R.

Affective and continuance commitment to the organization: evaluation of measures and analysis of concurrent and time lagged relations. Journal of Applied Psychology, v. 75, n. 6, p. 710-720, 1990.

MEYER, J. P.;

ALLEN, N. J.;

SMITH, C. A.

Commitment to organizations and occupations: extension and test of a three-component conceptualization. Journal of Applied Psychology, v. 78, n. 4, p. 538-551, 1993.

MOWDAY, R. T.;

PORTER, L. W.;

STEERS, R. M.

The measurement of organizational commitment. Journal of Vocational Behavior, v. 14, p. 224-247, 1979.

OLIVEIRA, A. de F.

Valores e ritos organizacionais como antecedentes do vínculo afetivo com a organização. Brasília, 1997. 143 f. Dissertação (Mestrado em Psicologia) - Instituto de Psicologia, Universidade de Brasília.

OLIVEIRA, A. de F.; GOMIDE JR., S.

Vínculos indivíduo-organização: um teste de dois modelos preditivos. In: CONGRESSO INTERNACIONAL DA ASSOCIAÇÃO BRASILEIRA DE ESTUDOS CANADENSES, 4., 1997, Uberlândia. Anais... [S.1.: s.n.], 1997. 
RITZER, G.;

TRICE, H. M.

An empirical study of Howard Becker's side-bet theory. Social Forces, v. 47, p. 475-479, 1969.

\section{SMIRCICH, L.}

Concepts of culture and organizational analysis. Administrative Science Quarterly, v. 28, p. 339-358, Sept. 1983.
WEINER, Y.

Commitment in organizations: a normative view. Academy Management Review, v. 7, p. 418-428, 1982.

WEINER, Y.;

VARDI, Y.

Relationships between job, organization, and career commitment and work outcomes: an integrate approach organizational behavior. Human Performance, v. 26, p. 81 96,1990. 